\title{
A NOTE ON THE SCHRÖDINGER MAXIMAL FUNCTION
}

\author{
J. BOURGAIN
}

\begin{abstract}
It is shown that control of the Schrödinger maximal function $\sup _{0<t<1}\left|e^{i t \Delta} f\right|$ for $f \in H^{s}\left(\mathbb{R}^{n}\right)$ requires $s \geq \frac{n}{2(n+1)}$
\end{abstract}

\section{INTRODUCTION}

Recall that the solution of the linear Schrödinger equation

$$
\left\{\begin{array}{l}
i u_{t}-\Delta u=0 \\
u(x, 0)=f(x)
\end{array}\right.
$$

with $(x, t) \in \mathbb{R}^{n} \times \mathbb{R}$ is given by

$$
e^{i t \Delta} f(x)=(2 \pi)^{-n / 2} \int e^{i\left(x \cdot \xi+t|\xi|^{2}\right)} \hat{f}(\xi) d \xi
$$

Assuming $f$ belongs to the space $H^{s}\left(\mathbb{R}^{n}\right)$ for suitable $s$, when does the almost convergence property

$$
\lim _{t \rightarrow 0} e^{i t \Delta} f=f \text { a.e. }
$$

hold? The problem was brought up in Carleson's paper [C] who proved convergence for $s \geq \frac{1}{4}$ when $n=1$. Dahlberg and Kenig [D-K] showed that this result is sharp. In higher dimension, the question of identifying the optimal exponent $s$ has been studied by several authors and our state of knowledge may be summarized as follows. For $n=2$, the strongest result to date appears in $\mathrm{L}$ and asserts (1.3) for $f \in H^{s}\left(\mathbb{R}^{2}\right), s>\frac{3}{8}$. More generally, for $n \geq 2$ (1.3) was shown to hold for $f \in H^{s}\left(\mathbb{R}^{n}\right), s>\frac{2 n-1}{4 n}$ (see [B] $)$.

In the opposite direction, for $n \geq 2$ the condition $s \geq \frac{n}{2(n+2)}$ was proven to be necessary (see [L-R] and also D-G] for a different approach based on pseudoconformal transformation). Here we show the following stronger statement.

Proposition 1. Let $n \geq 2$ and $s<\frac{n}{2(n+1)}$. Then there exist $R_{k} \rightarrow \infty$ and $f_{k} \in L^{2}\left(\mathbb{R}^{n}\right)$ with $\hat{f}_{k}$ supported in the annulus $|\xi| \sim R_{k}$, such that $\left\|f_{k}\right\|_{2}=1$ and

$$
\lim _{k \rightarrow \infty} R_{k}^{-s}\left\|\sup _{0<t<1}\left|e^{i t \Delta} f_{k}(x)\right|\right\|_{L^{1}(B(0,1))}=\infty .
$$

Date: September 20, 2016.

The author was partially supported by NSF grants DMS-1301619. 
There is some evidence the exponent $\frac{n}{2(n+1)}$ could be the optimal one, though limited to multi-linear considerations appearing in $[\mathrm{B}$. Of course, the $n=1$ case coincides with the $[\mathrm{D}-\mathrm{K}]$ result, while for $n=2$, the above Proposition leaves a gap between $\frac{1}{3}$ and $\frac{3}{8}$. It may be also worth to point out that for $n=2$, in some sense, our example fits a scenario where the arguments from [B] require the $s>\frac{3}{8}$ condition.

\section{An EXAMPLE}

Denote $x=\left(x_{1}, \ldots, x_{n}\right)=\left(x_{1}, x^{\prime}\right) \in B(0,1) \subset \mathbb{R}^{n}$. Let $\varphi: \mathbb{R} \rightarrow \mathbb{R}_{+}, \Phi: \mathbb{R}^{n-1} \rightarrow$ $\mathbb{R}_{+}$satisfy supp $\hat{\varphi} \subset[-1,1]$, supp $\hat{\Phi} \subset B(0,1), \hat{\varphi}, \hat{\Phi}$ smooth and $\varphi(0)=\Phi(0)=1$. Set $D=R^{\frac{n+2}{2(n+1)}}$ and define

$$
f(x)=e\left(R x_{1}\right) \varphi\left(R^{\frac{1}{2}} x_{1}\right) \Phi\left(x^{\prime}\right) \prod_{j=2}^{n}\left(\sum_{\frac{R}{2 D}<\ell_{j}<\frac{R}{D}} e^{i D \ell_{j} x_{j}}\right)
$$

where $\ell=\left(\ell_{2}, \ldots, \ell_{n}\right) \in \mathbb{Z}^{n-1}$. Hence

$$
\|f\|_{2} \sim R^{-\frac{1}{4}}\left(\frac{R}{D}\right)^{\frac{n-1}{2}} \text { and } \operatorname{supp} \hat{f} \subset[|\xi| \sim R] .
$$

Clearly, denoting $e(z)=e^{i z}$,

$e^{i t \Delta} f(x)=\iint \hat{\varphi}(\lambda) \hat{\Phi}\left(\xi^{\prime}\right)\left\{\sum_{\ell} e\left(\left(R+\lambda R^{\frac{1}{2}}\right) x_{1}+\left(\xi^{\prime}+D \ell\right) \cdot x^{\prime}+\left(R+\lambda R^{\frac{1}{2}}\right)^{2} t+\left|\xi^{\prime}+D \ell\right|^{2} t\right)\right\} d \lambda d \xi^{\prime}$.

Taking $|t|<\frac{c}{R},|x|<c$ for suitable constant $c>0$, one gets

$$
\begin{aligned}
\left|e^{i t \Delta} f(x)\right| & \sim\left|\int \hat{\varphi}(\lambda)\left\{\sum_{\ell} e\left(\lambda R^{\frac{1}{2}} x_{1}+D \ell \cdot x^{\prime}+2 \lambda R^{\frac{3}{2}} t+D^{2}|\ell|^{2} t\right)\right\} d \lambda\right| \\
& \sim \varphi\left(R^{\frac{1}{2}}\left(x_{1}+2 R t\right)\right)\left|\sum_{\ell} e\left(D \ell \cdot x^{\prime}+D^{2}|\ell|^{2} t\right)\right|
\end{aligned}
$$

Specify further $t=-\frac{x_{1}}{2 R}+\tau$ with $|\tau|<\frac{1}{10} R^{-\frac{3}{2}}$ in order to ensure that the first factor in (2.3) should be $\sim 1$. For this choice of $t$, the second factor becomes

$$
\begin{gathered}
\left|\sum_{\ell} e\left(D \ell \cdot x^{\prime}-\frac{D^{2}}{2 R}|\ell|^{2} x_{1}+D^{2}|\ell|^{2} \tau\right)\right|= \\
\prod_{j=2}^{n}\left|\sum_{\frac{R}{2 D}<\ell_{j}<\frac{R}{D}} e\left(\ell_{j} y_{j}+\ell_{j}^{2}\left(y_{1}+s\right)\right)\right|
\end{gathered}
$$

with

$$
y^{\prime}=D x^{\prime}(\bmod 2 \pi) \quad y_{1}=-\frac{D^{2}}{2 R} x_{1}(\bmod 2 \pi)
$$

and where $s=D^{2} \tau$ is subject to the condition

$$
|s| \lesssim D^{2} R^{-3 / 2}=R^{-\frac{n-1}{2(n+1)}} .
$$


We view $y=\left(y_{1}, y^{\prime}\right)$ as a point in the $n$-torus $\mathbb{T}^{n}$. Next, define the following subset $\Omega \subset \mathbb{T}^{n}$

$$
\Omega=\bigcup_{q \sim R^{\frac{n-1}{2(n+1)}}, a}\left\{\left(y_{1}, y^{\prime}\right) ;\left|y_{1}-2 \pi \frac{a_{1}}{q}\right|<c R^{-\frac{n-1}{2(n+1)}} \text { and }\left|y^{\prime}-2 \pi \frac{a^{\prime}}{q}\right|<c \frac{D}{R}\right\}
$$

with $a=\left(a_{1}, a^{\prime}\right)(\bmod q)$ and $\left(a_{1}, q\right)=1$.

Hence $|\Omega| \sim R^{\frac{n-1}{2(n+1)}} R^{n \frac{n-1}{2(n+1)}} R^{-\frac{n-1}{2(n+1)}}\left(\frac{D}{R}\right)^{n-1} \sim 1$ and we take $x \in B(0,1)$ for which $y$ given by (2.5) belongs to $\Omega$. Clearly this gives a set of measure at least $c_{1}>0$. We evaluate (2.4) for $y \in \Omega$. Let $q \sim R^{\frac{n-1}{2(n+1)}}$ and $\left(a_{1}, a^{\prime}\right)(\bmod q)$ satisfy the approximations stated in (2.7) and set $s=2 \pi \frac{a_{1}}{q}-y_{1}$ for which (2.6) holds. Clearly for $j=2, \ldots, n$, by the quadratic Gauss sum evaluation

$$
\begin{aligned}
\left|\sum_{\frac{R}{2 D}<\ell_{j}<\frac{R}{D}} e\left(\ell_{j} y_{j}+\ell_{j}^{2}\left(y_{1}+s\right)\right)\right| & \sim\left|\sum_{\frac{R}{2 D}<\ell_{j}<\frac{R}{D}} e\left(2 \pi \frac{a_{j}}{q} \ell_{j}+2 \pi \frac{a_{1}}{q} \ell_{j}^{2}\right)\right| \\
& \sim R^{\frac{1}{2(n+1)}}\left|\sum_{\ell_{j}=0}^{q-1} e\left(2 \pi \frac{a_{j}}{q} \ell_{j}+2 \pi \frac{a_{1}}{q} \ell_{j}^{2}\right)\right| \\
& \sim R^{\frac{1}{2(n+1)}} q^{\frac{1}{2}} \sim R^{\frac{1}{4}}
\end{aligned}
$$

and

$$
\text { (2.4) } \sim R^{\frac{n-1}{4}} \text {. }
$$

Recalling (2.2), we obtain for $x \in B(0,1)$ in a set of measure $c_{1}>0$ that

$$
\sup _{0<t<1} \frac{\left|e^{i t \Delta} f(x)\right|}{\|f\|_{2}} \gtrsim R^{\frac{n-1}{4}} R^{\frac{1}{4}}\left(\frac{D}{R}\right)^{\frac{n-1}{2}}=R^{\frac{n}{2(n+1)}} .
$$

The claim in the Proposition follows.

\section{REFERENCES}

[B] J. Bourgain, On the Schrödinger maximal function in higher dimension, Tr. Mat. Inst. Steklova 280 (2013). Ortogonalnye Ryady, Teoriya Priblizhenii i Smezhuye Voprosy, 5366; reprinted in Proc. Steklov Inst. Math. 280 (2013), no. 1, 46-60.

[C] L. Carleson, Some analytic problems related to statistical mechanics, Euclidean Harmonic Analysis (Proc. Sem., Univ. Maryland, College Park, Md. 1979), Lecture Notes in Math., Vol. 779, Springer, Berlin, 1980, pp. 5-45.

[D-G] C. Demeter, S. Guo, Schrödinger maximal function estimates via the pseudoconformal transformation, arXiv:1608.07640

[D-K] B.E.J. Dahlberg, C. E. Kenig, A note on the almost everywhere behavior of solutions to the Schrödinger equation, Harmonic Analysis (Minneapolis, Minn. 1981), Lecture Notes in Math., Vol. 908, Springer, Berlin, 1982, pp. 205-209.

[L] S. Lee, On pointwise convergence of the solutions to Schrödinger equations in $\mathbb{R}^{2}$, IMRN, Vol. 2006, 1-21.

[L-R] R. Lucà, K. Rogers, An improved necessary condition for the Schrödinger maximal estimate, available on arXiv. 
J. Bourgain, Institute for Advanced Study, Princeton, NJ 08540

E-mail address: bourgain@math.ias.edu 